\title{
Objetos de aprendizaje para la enseñanza de la física $^{*}$
}

\author{
José Antonio Gómez-Tejedor ${ }^{1}$ \\ ${ }^{1}$ ETS de Ingeniería del Diseño. Universitat Politècnica de València
}

\begin{abstract}
This paper describes several applets based on Easy Java Simulations. These applets are available as "learning objects" in the UPV RiuNet database. They are freely available so they can be used for any subject of physics from the UPV or any other institution. Through these learning objects, students can perform several lab sessions in the subject of Physics, or can perform simulations for displaying different physical phenomena. A special feature to note is that the applets are designed so that students can make measurements on the screen, similar to how they would in the physics laboratory of the subject. This allows to set out several lab sessions and tasks of the subject via the Internet thanks to these learning objects.
\end{abstract}

Keywords: Physics, Speed of sound, Rubens'tube, Standing waves, Elastic collision, Momentum and mechanical energy conservation, Converging lens, Ray diagram, Image formation.

\begin{abstract}
Resumen
Este trabajo describe una serie de applets basados en Easy Java Simulations. Estos applets están disponibles como "objetos de aprendizaje" en la base de datos Riunet de la UPV, y son accesibles en abierto, de modo que pueden ser utilizados por cualquier asignatura de física tanto de la UPV como de cualquier otra institución. Mediante estos objetos de aprendizaje, los alumnos pueden realizar diversas prácticas de laboratorio de la asignatura de Física, o pueden realizar simulaciones para la visualización de
\end{abstract}

${ }^{*}$ El autor de este trabajo agradece el apoyo del Instituto de Ciencias de la Educación de la UPV, programa de Equipos de Innovación y Calidad Educativa, por el apoyo al Equipo de Innovación en Metodologías Activas para el Aprendizaje de la Física (e-MACAFI), del cual formo parte, así como por subvencionar el proyecto PIME/2014/A/031/B que ha dado lugar a este trabajo. 
diferentes fenómenos físicos. Una particularidad a destacar es que los applets están diseñados para que los alumnos pueden realizar medidas sobre la pantalla del ordenador, de forma similar a como lo harían en el laboratorio de física de la asignatura. Esto permite plantear la realización de una serie de prácticas y tareas de la asignatura a través de Internet mediante estos objetos de aprendizaje.

Keywords: Física, Velocidad del sonido, Tubo de Rubens, Ondas estacionarias, Choque elástico, Conservación del momento lineal y de la energía mecánica, Lente convergente, Diagrama de rayos, Formación de imágenes.

\section{Introducción}

Los estudiantes que cursan asignaturas de Fundamentos Físicos de la Ingeniería o similares, deben de trabajar multitud de conceptos nuevos, algunos de los cuales bastante complicados de entender. Este trabajo describe una serie de animaciones interactivas de física basadas en Easy Java (Francisco Esquembre 2004; Christian y Francisco Esquembre 2007; Dormido y col. 2005), accesible a través de Internet, que el alumno puede utilizar para visualizar diferentes fenómenos físicos. Además, el alumno puede interaccionar con estas animaciones, de modo que dispone de una herramienta útil y versátil para facilitar su estudio individual. De esta forma, este trabajo se engloba en la tendencia actual de la aplicación de las nuevas tecnologías a metodologías activas de aprendizaje, para potenciar así el autoaprendizaje y el aprendizaje integral de los estudiantes (Gómez-Tejedor, Martínez y Vidaurre 2008; Gómez-Tejedor y G. 2009).

La idea de laboratorios virtuales y simulaciones basados en web no es nueva, ya en los años noventa aparecieron los primeros trabajos pioneros en esta línea, entre los que se puede citar los trabajos de Hoffman y col. 1994; Potter y col. 1996; Preis y col. 1997 entre otros. Este tema ha recibido mucha más atención en el últimos años debido a la implementación de nuevas tecnologías de enseñanza en el aula, y la rápida expansión de Internet. Actualmente, se pueden encontrar un gran número de laboratorios virtuales y simulaciones on-line, cubriendo temáticas muy diferentes: medida de dureza en metales (Hashemi, Chandrashekar y Anderson 2006), microbiología (Sancho y col. 2006), ingeniería de terremotos (Gao y col. 2005), aplicaciones medioambientales (Ascione y col. 2006), motores (Dias, Violato y Martins 2012), comunicación digital (Zhan, Porter y Morgan 2014), física (Chuang, Hwang y Tsai 2008), electrónica (I. A. Garcia, Pacheco y J. N. Garcia 2014) y simulación de circuitos electrónicos (Gómez-Tejedor, Martínez y Vidaurre 2008; Gómez-Tejedor y G. 2009; Bingol y Pacaci 2010; Zacharia y Jong 2014), por nombrar unos pocos de los muchos existentes, algunos de ellos pioneros en esta línea. Todas estas simulaciones han sido desarrollados en diferentes lenguajes de programación como Java, Matlab, LabView o Macromedia Flash.

Desde hace unos años, han ido apareciendo nuevas herramientas de desarrollo que no necesitan grandes conocimientos de programación, lo cual facilita en gran medida la creación de estas simulaciones, y abre nuevas posibilidades para todos aquellos 
profesores que no son expertos en programación. Por ejemplo, MatLab y Mathematica incorporan herramientas que permiten crear fácilmente aplicaciones web, pudiendo realizar diferentes simulaciones de procesos, en los que el usuario puede interactuar con la aplicación web. En esta línea podemos citar los trabajos de Savas y Erdal 2010; Andres Ramos-Paja, Ramirez Scarpetta y Martinez-Salamero 2010; Duque Grajales y col. 2009 entre muchos otros.

En este trabajo, se ha utilizado la herramienta Easy Java Simulations, desarrollada por Francisco Esquembre (F. Esquembre 2014), quien lo distribuye bajo Licencia Pública General de GNU (GPL de GNU), y que forma parte del proyecto Open Source Physics (Open Source Physics 2014).

Las simulaciones presentadas en este trabajo permiten visualizar diferentes procesos físicos. Los applets descritos en este trabajo se encuentran disponibles para su uso en el repositorio institucional de la Universitat Politècnica de València RiuNet como objetos de aprendizaje. En este trabajo se han seleccionado unos pocos de estos objetos de aprendizaje. Para una relación completa de dichos objetos, se puede consultar la base de datos del repositorio institucional de la Universitat Politècnica de València RiuNet, mediante la búsqueda por autor (Gómez-Tejedor 2014).

\section{Objetivos}

Los objetivos generales de esta propuesta son:

1. Preparar las clases de física: el alumnos puede utilizar las simulaciones para estudiar el fenómeno físico que se va a analizar en clase, ya bien sea una sesión teórica como una sesión de laboratorio.

2. Plantear tareas: el profesor puede utilizar los applets para plantear pequeñas tareas a los alumnos que pueden resolver de manera individual o en pequeños grupos.

3. Realizar prácticas de laboratorio que por razones de tiempo o de presupuesto no se puedan llevar a cabo en el laboratorio real.

Dado que los objetos de aprendizaje descritos en este trabajo abordan diferentes aspectos de la asignatura, se pueden plantear también una serie de objetivos concretos:

1. Estudiar el choque elástico de dos cuerpos en una dimensión.

2. Aplicar la conservación de energía y momento lineal en un choque elástico.

3. Visualizar el diagrama de rayos de una lente convergente.

4. Calcular la amplificación lateral, y la distancia a la que se forma la imagen en una lente convergente. 
5. Visualizar ondas estacionarias mediante un tubo de Rubens.

6. Calcular la velocidad del sonido en el aire.

\section{Descripción del trabajo}

En este trabajo se presentan una serie de simulaciones realizadas mediante la aplicación Easy Java Simulations (EJS). Estas simulaciones permiten estudiar diferentes conceptos teóricos de la asignatura de una manera práctica y visual. En concreto, se propone el estudio de los siguientes ejemplos de mecánica y óptica:

- Choque elástico. Conservación del momento lineal y de la energía cinética.

- Ondas estacionarias. Tubo de Rubens.

- Óptica geométrica: Diagrama de rayos para una lente convergente delgada.

\subsection{Choque elástico. Conservación del momento lineal y de la energía cinética}

Se dispone de dos vehículos, uno de ellos en reposo, y el otro con una velocidad inicial desconocida. El alumno puede seleccionar, mediante respectivas deslizaderas la masa de los dos cuerpos. El primero de ellos choca con el segundo, mediante un choque completamente elástico. Mediante un cronómetro situado en la parte superior de la pantalla, y una escala graduada en metros, el alumno puede medir posición y tiempo de los cuerpos para calcular sus velocidades.

A partir de estos datos, el alumno puede comprobar la ley de conservación de momento lineal, y de conservación de energía mecánica, teniendo en cuenta además que se considera despreciable el rozamiento de los cuerpos con el suelo.

A continuación se muestra un ejemplo de realización de una simulación. En ella se calcula la velocidad del centro de masas antes y después del choque, la velocidad primer vehículo antes del choque, y las velocidades de los dos vehículos después del choque.

Para calcular las velocidades antes del choque se muestran dos capturas de pantalla de la simulación, antes del choque de los dos cuerpos (Figura 1)

Teniendo en cuenta la posición del centro de masas, la posición del primer cuerpo, y el tiempo indicado por el cronómetro se puede calcular la velocidad del centro de masas,

$$
v_{c m}=\frac{200-150}{11,66-1,12}=4,74 \mathrm{~m} / \mathrm{s}
$$

Y la velocidad del primer vehículo: 


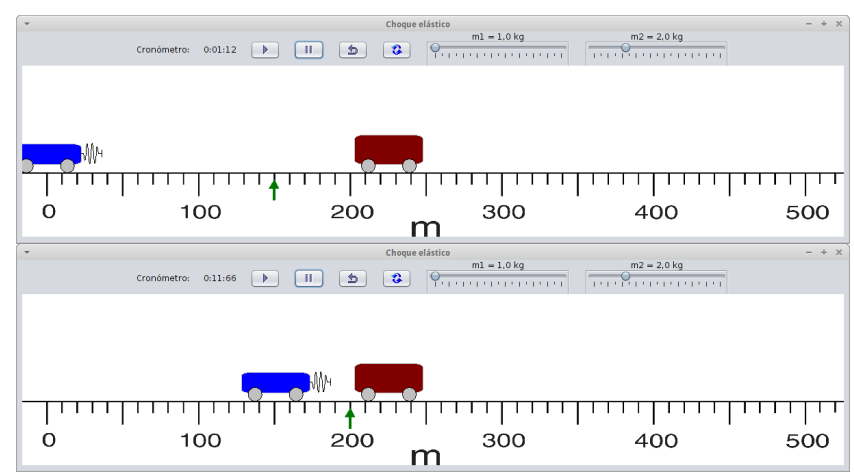

Fig. 1: Simulación antes y después del choque

$$
v_{1}=\frac{150-0}{11,66-1,12}=14,23 \mathrm{~m} / \mathrm{s}
$$

A partir de las posiciones del centro de masas y de los dos cuerpos, y de la medida del tiempo, el alumno puede calcular de forma análoga la velocidad del centro de masas, y las velocidades de los dos cuerpos después del choque.

A partir de los datos obtenidos, el alumno puede observar que, como era de esperar, la velocidad del centro de masas no cambia con el choque. Puede comprobar además la conservación del del momento lineal y al tratarse de un choque elástico, la conservación de la energía cinética:

En la toma de datos, el alumno puede encotrar pequeñas diferencias en los resultados debidas al error cometido en la determinación de la posición de los cuerpos en la escala graduada de la pantalla. El alumno deberá realizar tres medidas de cada magnitud, y calcular valores medios y desviación típica, de la misma manera que en una experiencia real en le laboratorio de física.

\subsection{Ondas estacionarias. Tubo de Rubens}

El Tubo de Rubens es un dispositivo que permite la visualización de ondas sonoras. Consiste en un tubo, que se llena de gas inflamable. En uno de los extremos del tubo se coloca un altavoz, y el otro permanece cerrado. Sobre el tubo se realizan una serie de pequeños orificios que permiten la salida del gas, prendiendo el gas a su salida por los orificios. En las zonas donde la amplitud de la onda sonora es mayor, la presión aumenta, y eso produce que la llama en ese punto sea más alta, y por el contrario donde la presión es menor la llama es más baja, o incluso desaparece cuando la presión externa es mayor que la presión en el interior del tubo, impidiendo la salida del gas por esos puntos. De esta forma, podemos visualizar de manera muy espectacular y llamativa las ondas sonoras que se propagan en el interior del tubo. El altavoz se 
conecta a un generador de funciones, que proporciona una señal sinusoidal, de modo que dentro del tubo tenemos la propagación de dicha onda sonora, junto con las ondas reflejadas en el extremo del tubo. A partir de la medida de la distancia entre nodos en la onda, y conocida la frecuencia del generador, podemos determinar de forma sencilla la velocidad del sonido en el tubo. En el Departamento de Física Aplicada de la ETS de Ingeniería del Diseño disponemos de un tubo de Rubens. Una de las características fundamentales del tubo de Rubens es su espectacularidad. Con esta simulación no se pretende sustituir dicho dispositivo, sino que intenta ser una ayuda para el profesor a la hora de introducir su funcionamiento. Permite que el profesor explique como van a realizar la medida sobre el simulador, antes de trabajar con el tubo de Rubens real, y permite que los alumnos entiendan mejor su funcionamiento.

A continuación se muestra un ejemplo de la medida de la velocidad del sonido en el interior del tubo. Para ello, en primer lugar el alumno debe de seleccionar una frecuencia de resonancia del tubo, para conseguir una onda estacionaria. En dicha situación, la separación entre dos nodos consecutivos es igual a la mitad de la longitud de onda de la señal que se propaga en el interior del tubo. Midiendo dicha distancia, y conociendo la frecuencia del generador, el alumno puede determinar la velocidad del sonido en el interior del tubo: $v=\lambda f$.

Para medir dicha distancia, disponemos de la información adicional de que la separación entre dos agujeros consecutivos del tubo, en este caso, es igual a $1 \mathrm{~cm}$. También se puede utilizar el cursor del ratón para medir distancias: pulsando sobre cualquier punto de la pantalla, en la esquina inferior izquierda, resaltado en amarillo aparecen las coordenadas $(x, y)$ del puntero del ratón. De esta forma, se puede medir la distancia de separación entre dos puntos cualquiera de la pantalla. Dichas coordenadas vienen dadas en mm.

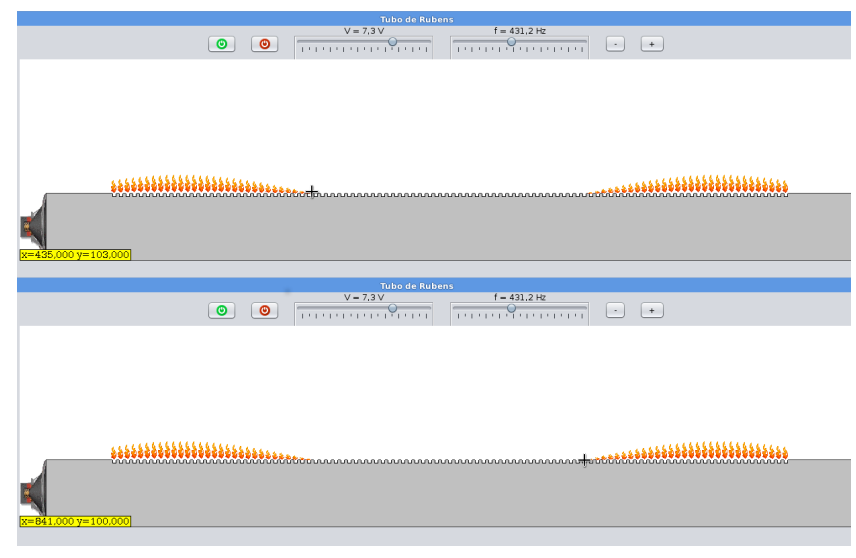

Fig. 2: Tubo de Rubens

En ejemplo, la separación entre los dos nodos consecutivos es de $841-435=406 \mathrm{~mm}$, de modo que la longitud de onda y la velocidad del sonido son respectivamente: 


$$
\begin{gathered}
\lambda=2 \cdot 0,406=0,812 \mathrm{~m} \\
v=\lambda f=0,812 \cdot 431,2=350,1 \mathrm{~m} / \mathrm{s}
\end{gathered}
$$

El alumno encontra?a diferencias en los valores observados en diferentes ejemplos debidas a los errores experimentales a la hora de medir las distancias sobre el tubo de Rubens, y a la hora de encontrar las frecuencias de resonancia de forma precisa. Dichas dificultades experimentales son las mismas con las que puede encontrar un alumno en el desarrollo de las medidas en un dispositivo real.

El alumno deberá realizar tres medidas de cada magnitud, y calcular valores medios y desviación típica, de la misma manera que en una experiencia real en le laboratorio de física.

\section{3 Óptica geométrica: Diagrama de rayos para una lente convergente delgada}

Este applet permite visualizar el diagrama de rayos de una lente convergente para la formación de la imagen. Además, el alumno puede realizar diversas medidas sobre la pantalla del ordenador: distancias focales, posición del objeto y de la imagen, tamaño del objeto y la imagen. La aplicación permite además variar la posición y tamaño del objeto, así como la distancia focal de la lente.

Al comenzar la aplicación, podemos ver una lente convergente, cuya distancia focal tiene un valor inicial aleatorio. Se puede observar también un objeto, así como la imagen formada por la lente de dicho objeto. Además se observa el diagrama de rayos correspondiente, donde se puede observar el rayo que pasa por el primer punto focal que se desvía hacia el infinito (paralelo al eje óptico), el rayo central, que no sufre ningunas desviación, y el rayo proveniente del infinito (paralelo al eje óptico) que pasa por el segundo punto focal de la lente. El primer y segundo punto focal de la lente aparecen indicado sobre el eje óptico mediante un punto rojo y uno azul respectivamente, y pueden cambiarse, simplemente pulsando con el puntero del ratón, y arrastrando a izquierda y derecha. El tamaño y posición del objeto también puede variarse de forma arbitraria, de la misma forma.

Conocida la posición del objeto, $s$, y la distancia focal de la lente, f, podemos calcular la posición de la imagen, $s^{\prime}$, mediante la ecuación:

$$
\frac{1}{s}+\frac{1}{s^{\prime}}=\frac{1}{f}
$$

Y el tamaño de la imagen viene dada por,

$$
y^{\prime}=-y \frac{s^{\prime}}{s}
$$




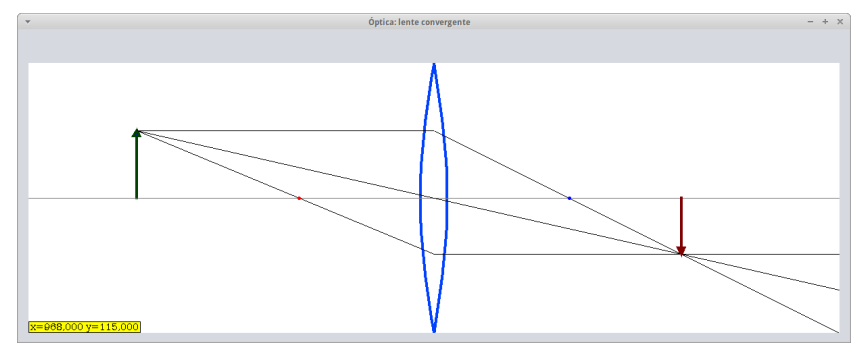

Fig. 3: Diagrama de rayos para una lente convergente delgada

En la figura 3 muestra un ejemplo de la imagen formada por la lente convergente de un objeto. Para medir cualquier distancia en la pantalla del ordenador, basta con pulsar sobre cualquier punto de la pantalla, y en la parte inferior izquierda aparece en color amarillo las coordenadas del punto en cuestión. Midiendo la posición de dos puntos, se puede calcular la distancia entre ambos puntos. Las distancias vienen dadas en mm.

En ese ejemplo, a partir de las medidas realizadas en la pantalla se obtiene $\mathrm{f}=200$ $\mathrm{mm}, \mathrm{s}=440 \mathrm{~mm}, \mathrm{y} \mathrm{y}=100 \mathrm{~mm}$. En la figura, se muestra a modo de ejemplo, las determinación de las coordenadas de uno de los puntos de la imagen ( $\mathrm{x}=968 \mathrm{~mm}$ y $\mathrm{y}=115 \mathrm{~mm}$ ). Del mismo modo, a partir de la medida de las coordenadas del resto de puntos, se obtienen todos los parámetros necesarios.

A partir de estos datos podemos calcular la posición de la imagen:

$$
s^{\prime}=\left(\frac{1}{f}-\frac{1}{s}\right)^{-1}=\left(\frac{1}{200}-\frac{1}{440}\right)^{-1}=367 \mathrm{~mm}
$$

Y el tamaño de la imagen viene dada por,

$$
y^{\prime}=-y \frac{s^{\prime}}{s}=-100 \frac{367}{440}=-83,3 \mathrm{~mm}
$$

Por otro midiendo sobre la pantalla del ordenador, obtenemos $s^{\prime}=365 \mathrm{~mm}$ y $h^{\prime}=$ $-87 \mathrm{~mm}$ (imagen invertida). Vemos que hay unas pequeñas diferencias entre el valor obtenido en la medida directa sobre la pantalla, y en el resultado calculado. Esto es debido a los errores cometidos en la toma de datos sobre la pantalla del ordenador, de la misma forma que ocurriría en el laboratorio en un experimento real.

El alumno deberá realizar tres medidas de cada magnitud, y calcular valores medios y desviación típica, de la misma manera que en una experiencia real en le laboratorio de física. 


\section{Resutados}

Los alumnos que han trabajado con estas simulaciones, muestran un alto grado de satisfacción. Para ellos, ha sido una herramienta útil en su proceso de aprendizaje, y la utilización de estas simulaciones les ha ayudado a entender los conceptos de la asignatura ilustrados en la simulación. Respecto a los profesores que han utilizado estas simulaciones, manifiestan que es una herramienta que facilita el proceso de enseñanza, y su versatilidad les permite su utilización en diferentes momentos del curso. Estas simulaciones pueden también ser utilizadas en aquellos casos en los que el alumno tiene alguna dificultad para acudir al laboratorio, como prácticas de la asignatura. En esta situación, el principal problema con el que se encuentran los alumnos es el tratamiento de datos mediante la hoja de cálculo. En ese caso sería conveniente complementar esta actividad con la realización de una sesión presencial para que el alumno aprenda a manejar la hoja de cálculo, y la utilización de tutorías on-line para resolver las dudas que puedan surgir en la realización de la práctica y el tratamiento de los datos.

\section{Conclusiones}

Como conclusión final, este trabajo permite estudiar de manera práctica diferentes conceptos de la asignatura de Física mediante cualquier ordenador conectado a Internet. Este trabajo se puede llevar a cabo de forma individual o en pequeños grupos, y sin un horario prefijado. Esto permite a los estudiantes regular su proceso de aprendizaje, invirtiendo el tiempo que sea necesario en cada sesión. Estas animaciones se pueden plantear también para la realización de prácticas de laboratorio. Se podría argumentar que el uso de un entorno virtual no es idéntico a la utilización de dispositivos reales en un laboratorio de física. Sin embargo, no se pretende sustituir las prácticas reales, sino que su objetivo es combinar la realización de prácticas virtuales con la realización de prácticas presenciales en el laboratorio. La realización virtual de un experimento no puede en ningún caso sustituir a la realización real de la práctica en el laboratorio.

\section{Agradecimientos}

El autor de este trabajo agradece el apoyo del Instituto de Ciencias de la Educación de la UPV, programa de Equipos de Innovación y Calidad Educativa, por el apoyo al Equipo de Innovación en Metodologías Activas para el Aprendizaje de la Física (e-MACAFI), del cual formo parte, así como por subvencionar el proyecto PIME/2014/A/031/B que ha dado lugar a este trabajo. 


\section{Referencias bibliográficas}

Andres Ramos-Paja, C., J. M. Ramirez Scarpetta y L. Martinez-Salamero (2010). "Integrated Learning Platform for Internet-Based Control-Engineering Education". English. En: IEEE TRANSACTIONS ON INDUSTRIAL ELECTRONICS 57.10, 3284-3296. ISSN: 0278-0046. DOI: \{10.1109/TIE. 2010.2043033\}.

Ascione, I. y col. (2006). "A Grid Computing Based Virtual Laboratory for Environmental Simulations". En: Euro-Par 2006 Parallel Processing. Ed. por WolfgangE. Nagel, WolfgangV. Walter y Wolfgang Lehner. Vol. 4128. Lecture Notes in Computer Science. Springer Berlin Heidelberg, págs. 1085-1094. ISBN: 978-3-54037783-2. DOI: 10.1007/11823285_114.

Bingol, O. y S. Pacaci (2010). "A virtual laboratory for fuzzy logic controlled DC motors". English. En: INTERNATIONAL JOURNAL OF THE PHYSICAL SCIENCES 5.16, 2493-2502. ISSN: 1992-1950.

Christian, Wolfgang y Francisco Esquembre (2007). "Modeling Physics with Easy Java Simulations". En: The Physics Teacher 45.8, págs. 475-480. DOI: http: //dx.doi.org/10.1119/1.2798358.

Chuang, Shih-Chyueh, Fu-Kwun Hwang y Chin-Chung Tsai (2008). "Students' Perceptions of Constructivist Internet Learning Environments by a Physics Virtual Laboratory: The Gap between Ideal and Reality and Gender Differences". En: CyberPsychology \& Behavior 11.2, págs. 150-156. ISSN: 1094-9313. DOI: 10.1089/ cpb. 2007.0024.

Dias, J. N., G. O. Violato y C. A. Martins (2012). "Dynamic model of a twostroke glow engine from experimental data". English. En: PROCEEDINGS OF THE INSTITUTION OF MECHANICAL ENGINEERS PART G-JOURNAL OF AEROSPACE ENGINEERING 226.G12, 1502-1512. ISSN: 0954-4100. DOI: $\{10.1177 / 0954410011426371\}$.

Dormido, S. y col. (2005). "Adding interactivity to existing Simulink models using Easy Java Simulations". En: Decision and Control, 2005 and 2005 European Control Conference. CDC-ECC '05. 44th IEEE Conference on, págs. 4163-4168. DOI: $10.1109 /$ CDC.2005.1582815.

Duque Grajales, J. E. y col. (2009). "System for Processing and Simulation of Brain Signals". English. En: 2009 IEEE LATIN-AMERICAN CONFERENCE ON COMMUNICATIONS (LATINCOM 2009). Ed. por Velasquez, CE. IEEE Latin-American Conference on Communications, Medellin, COLOMBIA, SEP 10-11, 2009. IEEE Commun Soc. 345 E 47TH ST, NEW YORK, NY 10017 USA: IEEE, 195-200. ISBN: 978-1-4244-4387-1. 
Esquembre, F. (2014). EasyJava Simulations. URL: http://www.um.es/fem/Ejs/.

Esquembre, Francisco (2004). "Easy Java Simulations: a software tool to create scientific simulations in Java". En: Computer Physics Communications 156.2, págs. 199-204. ISSN: 0010-4655. DOI: http://dx.doi.org/10.1016/S0010-4655(03)00440-5.

Gao, Y. y col. (2005). "Java-powered virtual laboratories for earthquake engineering education". En: Computer Applications in Engineering Education 13.3, págs. 200-212. ISSN: 1099-0542. DOI: 10.1002/cae.20050.

Garcia, I. A., C. L. Pacheco y J. N. Garcia (2014). "Enhancing education in electronic sciences using virtual laboratories developed with effective practices". English. En: COMPUTER APPLICATIONS IN ENGINEERING EDUCATION 22.2, 283-296. ISSN: 1061-3773. DOI: \{10.1002/cae.20554\}.

Gómez-Tejedor, J.A. (2014). Plan Docencia en Red. Laboratorios virtuales. URL: http: //riunet . upv . es /handle/10251/11259/browse? authority=171609\&type= author.

Gómez-Tejedor, J.A. y Moltó. G. (2009). Technologies Shaping Instruction and Distance Education: New Studies and Utilizations. Chapter \%: Online Learning of Electrical Circuits through a Virtual Laboratory. Ed. por Dr. Mahbubur Rahman Syed. IGI Global.

Gómez-Tejedor, J.A., G.M. Martínez y C.B. Vidaurre (2008). "An Online Virtual Laboratory of Electricity". En: International Journal of Distance Education Technologies 6.2, págs. 21-34. DOI: 10.4018/jdet.2008040102.

Hashemi, J., N. Chandrashekar y E. E. Anderson (2006). "Design and Development of an Interactive Web-based Environment for Measurement of Hardness in Metals: a Distance Learning Tool". En: International Journal of Engineering Education 22.5, págs. 993-1002.

Hoffman, C. M. y col. (1994). "SoftLab?A virtual laboratory for computational science". En: Mathematics and Computers in Simulation (MATCOM) 36.4, págs. 479-491.

Open Source Physics (2014). URL: http://www.opensourcephysics.org/.

Potter, C. y col. (1996). "EVAC: a virtual environment for control of remote imaging instrumentation". En: Computer Graphics and Applications, IEEE 16.4, págs. 62-66. ISSN: 0272-1716. DOI: 10.1109/38.511856.

Preis, K. y col. (1997). "A virtual electromagnetic laboratory for the classroom and the WWW". En: IEEE Transactions on Magnetics 33, págs. 1990-1993. DOI: $10.1109 / 20.582690$. 
Sancho, P. y col. (2006). "A Blended Learning Experience for Teaching Microbiology". En: American Journal of Pharmaceutical Education 70.5, pág. 120.

Savas, K. y H. Erdal (2010). "Automatic control simulation environment system (ACSES) designed as a virtual tool for control education". English. En: INNOVATION AND CREATIVITY IN EDUCATION. Ed. por Uzunboylu, H. Vol. 2. Procedia Social and Behavioral Sciences 2. 2nd World Conference on Educational Sciences (WCES-2010), Bahceschir Univ, Istanbul, TURKEY, FEB 04-08, 2010. SARA BURGERHARTSTRAAT 25, PO BOX 211, 1000 AE AMSTERDAM, NETHERLANDS: ELSEVIER SCIENCE BV, 5233-5237. DOI: $\{10.1016 /$ j.sbspro.2010.03.851\}.

Zacharia, Z. C. y T. de Jong (2014). "The Effects on Students' Conceptual Understanding of Electric Circuits of Introducing Virtual Manipulatives Within a Physical Manipulatives-Oriented Curriculum". English. En: COGNITION AND INSTRUCTION 32.2, 101-158. ISSN: 0737-0008. DOI: $\{10.1080 / 07370008.2014$. 887083\}.

Zhan, W., J. R. Porter y J. A. Morgan (2014). "Experiential Learning of Digital Communication Using LabVIEW". English. En: IEEE TRANSACTIONS ON EDUCATION 57.1, 34-41. ISSN: 0018-9359. DOI: \{10.1109/TE.2013.2264059\}. 